\title{
DETECTION OF ACQUIRED MULTIDRUG-RESISTANT TUBERCULOSIS AND EXTENSIVELY DRUG- RESISTANT TUBERCULOSIS IN AND AROUND KOLKATA IN RECENT PAST
}

\author{
C. P. Battacharya ${ }^{1}$, T. K. Battacharya ${ }^{2}$
}

${ }_{1}^{1}$ Microbiologist, Bengal Tuberculosis Association, Kolkata.

${ }^{2}$ Associate Professor, Department of Microbiology, MGM Medical College, LSK Hospital, Kishanganj.

\begin{abstract}
BACKGROUND
ABSTRACT

Nowadays tuberculosis is a serious global problem. There is an estimated 8-10 million new cases of tuberculosis worldwide with 23 million deaths occurring in each year. In many parts of the world, including India, tuberculosis is the leading cause of death from any one infectious agent. It has been further estimated that tuberculosis is directly responsible for an estimated $7 \%$ of all deaths and $26 \%$ of all preventable deaths throughout the world.

Aim- To find the antibiotic susceptibility patterns of isolates of Mycobacterium tuberculosis from different specimens in and around Kolkata after introduction of RNTCP.

Objective- To study the antitubercular drug-resistant pattern of the M. tuberculosis isolates in urban population.
\end{abstract}

\section{MATERIALS AND METHODS}

A prospective observational study was carried out in the laboratory of the institution. All data regarding patient characteristics, disease characteristics, along with previous treatment and the antibiotic sensitivity pattern of the isolates were prospectively recorded from April 2002 to June 2008. During this period, 461 of 1207 culture-positive cases were tested. Data were further analysed.

\section{RESULTS}

Out of 461 isolates of M. tuberculosis, there were 232 cases of multidrug-resistant TB (MDR-TB), the percentage being $54.4 \%$. Total number of extensively drug-resistant TB (XDR-TB) cases were 9, the percentage being $1.9 \%$. There was a steady increase in frequency of MDR - isolates with ongoing years.

\section{CONCLUSION}

The study demonstrates the antitubercular drug-resistant pattern of the M. tuberculosis isolates in mostly urban population who were treated with various combination of first-line antitubercular drugs including the drug regimen prescribed by RNTCP. It will help us in our efforts of controlling drug resistance by modifying treatment regimen and other lacunae in the delivery system.

\section{KEYWORDS}

MDR-TB, XDR-TB, Antibiotic Susceptibility, BACTEC Radiometric Method, Ziehl-Neelsen Staining Method.

HOW TO CITE THIS ARTICLE: Battacharya CP, Battacharya TK. Detection of acquired multidrug-resistant tuberculosis and extensively drug-resistant tuberculosis in and around Kolkata in recent past. J. Evolution Med. Dent. Sci. 2017;6(90):6312-6315, DOI: $10.14260 /$ jemds/2017/1373

\section{BACKGROUND}

Nowadays tuberculosis is a serious global problem. There is an estimated 8-10 million new cases of tuberculosis worldwide with 2-3 million deaths occurring in each year. In many parts of the world, including India, tuberculosis is the leading cause of death from any one infectious agent. It has been further estimated that tuberculosis is directly responsible for an estimated $7 \%$ of all deaths and $26 \%$ of all preventable deaths throughout the world.(1,2) The situation has become further complicated due to treatment failure and emergence of drug-resistant tuberculosis. In 2013, there may have emerged multidrug-resistant TB (MDR-TB) in an estimated 480,000 cases.

'Financial or Other Competing Interest': None.

Submission 04-08-2017, Peer Review 04-11-2017,

Acceptance 11-11-2017, Published 20-11-2017.

Corresponding Author:

Dr. C. P. Battacharya,

Bengal Tuberculosis Association,

PO-Entally 24,

Dr. Sundari Mohan Avenue,

Kolkata-700014.

E-mail: chandika.prasun@gmail.com

DOI: $10.14260 /$ jemds $/ 2017 / 1373$

At present, we cannot identify more than $72.0 \%$ of these resistant cases due to lack of culture and drug-susceptibility testing capability in most settings worldwide. On an average, $60 \%$ of all MDR-TB cases are in India, China, the Russian Federation, Pakistan, and Ukraine. From 2006 and onwards, 100 countries, including the United States have started reporting cases of extensively drug-resistant TB (XDR-TB). In these cases, MDR-TB shows additional resistance to the second-line antitubercular drugs. There is an apprehension that about $10 \%$ of MDR-TB cases worldwide are XDR-TB, but the vast majority of XDR cases remain beyond diagnosis. Recently, cases resistant to all anti-TB drugs have been reported from countries like India, Italy, and Iran. $(3,4)$

Drug-resistant $M$. tuberculosis arises by spontaneous point mutation in the mycobacterial genome. This process occurs at a low but predictable rates $\left(10^{-7}\right.$ to $10^{-10}$ for the concerned drug). As there is no cross-resistance among the commonly used antitubercular drugs, the probability that a particular strain will become resistant to two drugs simultaneously, is the product of the probabilities of resistance to each drug and thus is low. Drug-resistant tuberculosis may be either primary or acquired. In primary drug resistance, the strain of $M$. tuberculosis develops resistance in a patient who has not been treated previously. 
Acquired resistance always develops during treatment. The development of drug-resistant TB is a reflection of the system failure - the system which consists of physicians, inadequate drug delivery mechanisms and patients' ignorance coupled with poverty.

In primary drug resistance, the patient is infected from the beginning by a drug-resistant strain. In acquired resistance, it develops while the patient is under treatment with an inappropriate regimen. In most of Latin America, Western Europe, North America and the Persian Gulf States, rates of primary resistance are generally low and isoniazid resistance is most common. MDR-TB is an ever-increasing grave problem in some regions of the world, especially in the states of the former Soviet Union and some countries of Asia. In India, we have only subnational data.(3)

By definition, MDR-TB are resistant at least to rifampicin and isoniazid; while XDR-TB is due to MDR strains that are resistant to all fluoroquinolones and to at least one of the three second-line injectable agents (amikacin, kanamycin and capreomycin). Acquired resistance varies from region to region, from state to state, even from one metropolis to other. Therefore, area based studies regarding MDR-TB and XDR-TB has become paramount importance in controlling and treating such patients.

However, drug susceptibility tests for mycobacteria are technically challenging, expensive and time-consuming. Moreover, the facility is not available except in a few centres. The situation is further complicated due to non-producibility of the results because of slow growth kinetics of these bacteria and variable standardisation methods. In recent times, these lacunae of drug susceptibility studies have largely been overcome by using automated systems.

\section{MATERIALS AND METHODS}

A prospective observational study was carried during the period April 2002 to June 2008, 4596 specimens were received out of which the number of sputum samples were 3434 (75\%). Extrapulmonary specimens included cerebrospinal fluid (298), lymph nodes and other tissues (85), urine (115), pleural fluid (128), peritoneal fluid (150), pus (145) and bronchopulmonary lavage (240); total numbers being 1161 . The patients of the present study belong mainly to middle to lower socioeconomic status and mostly come from Kolkata and neighbouring districts. Based on prescriptions and history taking, it was found that they all were treated with various combinations of first-line drugs, e.g. Streptomycin (SM), isoniazid (INH), rifampicin (RMP), ethambutol (EMB) and pyrazinamide (PZA) for at least 2-3 years. INH and RMP were present in all the combinations.

They were sent for culture followed by drug sensitivity test for they were not responding to treatment as suspected either clinically and/or radiologically or evidenced bacteriologically by repeated smear positivity. Out of these 4596 cases, 1207 (26\%) were culture positive. However, only those culture-positive cases were tested whose drug sensitivity were sought by clinicians. After performing NAP test and thereby excluding non-tubercular mycobacteria (NTM) among them - 461 cases were selected for drug susceptibility -whose results are given in Table 2 . Unfortunately, due to non-availability of the $12 \mathrm{~B}$ vials, the study was discontinued. All specimens were smeared on clean scratch-free new glass slides and stained by carbol fuchsin, the classic Z-N stain, which requires heating. Smears stained with Z-N stain were examined at $1000 \times$ magnification (oil immersion). Results were reported after viewing 100 fields and included a statement indicating whether the smear was prepared directly from specimens or after decontamination and concentration. The method of reporting was the guideline as stated by WHO clinical manual for South-East Asia. All potentially contaminated specimens such as sputum, urine were liquefied, decontaminated, and concentrated by the $\mathrm{N}$ acetyl-L-cysteine (NALC) - Sodium hydroxide method and finally centrifuged at 3000 to $3600 \mathrm{X}$ g for 15 minutes.(5)

Specimens were inoculated into the BACTEC $12 \mathrm{~B}$ broth (Becton - Dickinson, USA). Using a sterile syringe $0.5 \mathrm{~mL}$ of the well-mixed specimen was added.

Specimens were inoculated into the BACTEC 12B broth (Becton-Dickinson, USA). Using a sterile syringe $0.5 \mathrm{~mL}$ of the well mixed specimen was added to the medium. Prior to specimen inoculation $0.1 \mathrm{~mL}$ of PANTA supplement (polymyxin B $50 \mu \mathrm{g} / \mathrm{mL}$, amphotericin B $5 \mu \mathrm{g} / \mathrm{mL}$, nalidixic acid $20 \mu \mathrm{g} / \mathrm{mL}$, trimethoprim $5 \mu \mathrm{g} / \mathrm{mL}$ and azlocillin 10 $\mu \mathrm{g} / \mathrm{mL}$ ) was added to each BACTEC $12 \mathrm{~B}$ vial. The media were incubated at $37^{\circ} \mathrm{C}$ for 6 weeks. The vials were tested every two days for the first 2 weeks and once in a week thereafter for growth. BACTEC vials showing a growth index (GI) equal to or more than 10 were read daily until the value of GI attained $50-100$. Growth index is a quantitative expression of radioactivity of $14 \mathrm{CO}_{2}$ - produced by the mycobacteria after metabolising 14C - containing Palmitic acid present in the medium as a metabolite. It is expressed in terms of numbers on a scale ranging from 0 to 999 . The growth was subjected to ZN stain and Gram stain. The vials remaining negative for 6 weeks, were discarded.(6)

The BACTEC mycobacterial cultures were tested by the BACTEC NAP (p-nitro- $\alpha$-acetyl-amino- $\beta$-hydroxypropiophenone) to confirm M. tuberculosis by differentiating it from non-tuberculous mycobacteria (NTM).(6,7) The NAP vial was inoculated with $1.0 \mathrm{~mL}$ of the culture from a positive $12 \mathrm{~B}$ vial. Both the test and control vials were further incubated at $37^{\circ} \mathrm{C}$ and the GI of both vials were read. The isolates were identified as $\mathrm{M}$. tuberculosis complex when the NAP vial showed two consecutive significant decreases in GI or slight increase in first two days followed by a decrease or no increase.

Once identified as an isolate of M. tuberculosis complex by NAP test, the original culture positive vial was incubated further till it attained a GI of $500-800$. This culture was used for susceptibility test. If the GI was more than 800 , the growth was diluted 1: 2 in the BACTEC diluting fluid prior to susceptibility testing. From the stock solutions of the primary drugs, $0.1 \mathrm{~mL}$ was added to four different $12 \mathrm{~B}$ vials to achieve final concentrations of Streptomycin $2 \mu \mathrm{g} / \mathrm{mL}$, Isoniazid 0.1 $\mu \mathrm{g} / \mathrm{mL}$, Rifampicin $2 \mu \mathrm{g} / \mathrm{mL}$ and Ethambutol $2.5 \mu \mathrm{g} / \mathrm{mL}, 0.1$ $\mathrm{mL}$ of the well-mixed culture was added to each of these vials. The second-line drugs (capreomycin $2.5 \mu \mathrm{g} / \mathrm{mL}$, cycloserine $70 \mu \mathrm{g} / \mathrm{mL}$, ethionamide $2.5 \mu \mathrm{g} / \mathrm{mL}$, kanamycin 2.5 microgram $/ \mathrm{mL}$, ciprofloxacin $1 \mu \mathrm{g} / \mathrm{mL}$ ) were procured from the manufacturer in pure chemical form. They are constituted and used in the concentrations mentioned above as per manufacturer's (BD) recommendation. The control vial was, however, inoculated with $0.1 \mathrm{~mL}$ of the culture, diluted $1: 100$. This method is equivalent to the proportion method, done by using Lowenstein-Jensen (LJ) media, which detects $1.0 \%$ or 
more of the drug-resistant population. Both the test and control vials were incubated at $37^{\circ} \mathrm{C}$ and examined daily for a minimum of 5 days and a maximum of 12 days. On the day the control vial reached a GI value of 30 or more, the difference in GI values from the previous, designated as $\triangle$ GI was less than the control vial by $10 \%$, it was reported as sensitive.(6)

This is to be noted that CDC recommends that drug susceptibility test results should be provided with 28 days of specimen receipt in the laboratory, a turnaround time that currently can be achieved only with the BACTEC TB system.(8)

The second-line drugs were procured from the manufacturer in pure chemical form and used e.g. Capreomycin $(2.5 \mu \mathrm{g} / \mathrm{mL})$, cycloserine $(70 \mu \mathrm{g} / \mathrm{mL})$, ethionamide $(2.5 \mu \mathrm{g} / \mathrm{mL})$, kanamycin $(2.5 \mu \mathrm{g} / \mathrm{mL})$ and ciprofloxacin $(1 \mu \mathrm{g} / \mathrm{mL})$.

\section{RESULTS}

A total of 461 strains of M. tuberculosis were selected for drug susceptibility from April 2002 to June 2008. Among the 461 cases; isolates from male patients were 295 and the number of female cases were 166. Most isolates belonged to age groups between 26 yrs. to 50 yrs. In children the most often clinical specimens were that of cerebrospinal fluid. Age wise distribution of cases has been shown in Table 1 .

\begin{tabular}{|c|c|c|c|c|c|c|c|c|c|}
\hline \multirow{2}{*}{\multicolumn{2}{|c|}{$\begin{array}{c}\text { - } 5 \text { yrs. } \\
\text { Total } \\
\text { (n) } 27\end{array}$}} & \multicolumn{4}{|c|}{\begin{tabular}{|l|l|l}
$6-15$ & yrs. & $16-25$ yrs \\
\end{tabular}} & \multirow{2}{*}{\multicolumn{2}{|c|}{\begin{tabular}{|c|}
$26-50$ yrs. \\
Total \\
(n) 201
\end{tabular}}} & \multirow{2}{*}{\multicolumn{2}{|c|}{$\begin{array}{c}>50 \text { yrs } \\
\text { Total } \\
\text { (n) } 80\end{array}$}} \\
\hline & & \multicolumn{2}{|c|}{$\begin{array}{l}\text { Total } \\
\text { (n) } 41\end{array}$} & \multicolumn{2}{|c|}{$\begin{array}{c}\text { Total } \\
\text { (n) } 112\end{array}$} & & & & \\
\hline 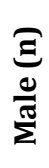 & 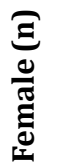 & 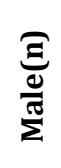 & $\frac{\Xi}{\frac{0}{\pi}}$ & $\underset{\frac{0}{\pi}}{\Xi}$ & 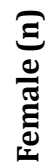 & $\underset{\substack{0 \\
\frac{\pi}{2}}}{\Xi}$ & 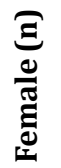 & $\underset{\frac{0}{\pi}}{\Xi}$ & 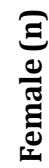 \\
\hline 20 & 7 & \begin{tabular}{|l|}
26 \\
\end{tabular} & 15 & 71 & 41 & 127 & 74 & 51 & 29 \\
\hline & & & & & & & & & \\
\hline
\end{tabular}

All the 461 isolates from BACTEC $12 \mathrm{~B}$ belonged to the M. tuberculosis complex based on the NAP differentiation test. The average duration required to detect growth by radiometric method was 2 weeks. All the isolates were subjected to drug sensitivity tests and number of MDR- and XDR - strains were identified by the definition already stated. Out of 461 cases, 251 cases were found to be MDR-TB strains. The percentage calculated was $54.4 \%$. There was a clear tendency of gradually increasing trend of MDR isolates in the subsequent years. However, the total number of XDR-TB cases were 9; the percentage being $1.9 \%$. The prevalence of MDR and XDR - TB strains has been depicted in the Table 2.

\begin{tabular}{|c|c|c|c|}
\hline Year & $\begin{array}{c}\text { Total no. of } \\
\text { Culture-Positive } \\
\text { Cases (n=461) }\end{array}$ & $\begin{array}{c}\text { Total no. of } \\
\text { MDR-strains } \\
(\mathbf{n = 2 5 1})\end{array}$ & $\begin{array}{c}\text { Total no. of } \\
\text { XDR-strains } \\
\text { (n=9) }\end{array}$ \\
\hline $\begin{array}{c}2002 \\
\text { (from April) }\end{array}$ & 23 & $16(69.0 \%)$ & 01 \\
\hline 2003 & 72 & $35(48.6 \%)$ & 01 \\
\hline 2004 & 89 & $41(46.0 \%)$ & 02 \\
\hline 2005 & 84 & $47(55.9 \%)$ & 02 \\
\hline 2006 & 91 & $47(51.6 \%)$ & 00 \\
\hline 2007 & 75 & $46(61.3 \%)$ & 03 \\
\hline $\begin{array}{c}2008 \\
\text { (up to June) }\end{array}$ & 27 & $19(70.3 \%)$ & 00 \\
\hline \multicolumn{2}{|c|}{ Table 2: Prevalence of MDR- and XDR-TB strains } \\
\hline
\end{tabular}

\section{DISCUSSION}

The study was carried out to find the prevalence of drugresistant $M$. tuberculosis in the treatment failure cases in the urban areas, mostly in Kolkata and surrounding zones. All the 461 isolates belonged to the $\mathrm{M}$. tuberculosis complex as identified by the NAP differentiation test. The drug susceptibility test was performed by the BACTEC radiometric method for all isolates of M. tuberculosis. The results were available within 3 weeks after identification of mycobacterial growth in the $12 \mathrm{~B}$ medium by $\mathrm{Z}-\mathrm{N}$ staining. The drug susceptibility patters suggest that there has been an alarming level of drug resistance among $\mathrm{M}$. tuberculosis strains as because out of 461 cases there were 251 cases of MDR-TB. The percentage calculated was $54.4 \%$. This very high percentage of MDR-TB strains was due to, apart from tertiary nature of the institute with referral bias, irregular treatment; irrational drug combination; inadequate doses of antitubercular drugs and shorter duration of therapy. Among MDR-TB strains, single - drug resistance recorded was with INH $75 \%$ and RIF was observed in $61 \%$ cases. There is another disturbing trend of a steady increase of MDR from $2003-2008$.

This study also reveals the advantage of using BACTEC system in isolation and drug sensitivity testing of suspected tuberculosis cases because of the rapidity of the system. A turnaround time of 28 days for the isolation and the drug susceptibility test has been achieved in almost all the cases. Therefore, the BACTEC 12B medium provides a tremendous advantage over any other procedures apart from molecular methods in terms of its rapidity in growth detection, increased sensitivity and a lower rate of specimen lost due to contamination. ${ }^{(9)}$ Moreover, automated radiometric procedure carries the potential of introducing standardisation in the mycobacteriology laboratory.(10)

There is one important observation in our present study. The total number of XDR- cases was only 9. The percentage calculated was $1.95 \%$. if we calculate the percentage incidence of XDR-strain among MDR-strain (a total of 232 cases), the value comes to be as $3.4 \%$. This tendency clearly indicates a future disaster among the residents in and around Kolkata.

However, the biggest hurdle associated with the case of BACTEC system in our country is the initial investment and the recurrent costs that are involved. By facilitating early diagnosis and drug susceptibility, the BACTEC method may prove to be cost-effective in a population with a high prevalence of tuberculosis. The importance of rapid identification and drug sensitivity pattern of mycobacteria for the management of tuberculosis outweighs the problems encountered due to cost constraints.

To control tuberculosis, the most important thing is to prevent the disease. As per CDC guidelines in 1988, there are four general strategies for controlling tuberculosis. The most important is early detection and adequate treatment of persons with infections tuberculosis. The second strategy entails identification and treatment of individuals with noncontiguous tuberculosis. The third strategy involves creation of a safe environment in situations where the risk of transmitting infection is high. The fourth strategy is vaccination with the BCG vaccine. In high TB prevalence countries, WHO recommends BCG for all children (according to standard programme) except children with symptoms of 
HIV disease/AIDS. Drug-resistant TB can be prevented by adherence to the principles of sound therapy; the inclusion of at least two bactericidal drugs to which the organism is susceptible; the use of fixed-drug-combination products, and the verification that patients complete the prescribed course.(5) There is also an urgent need to carry out the drug susceptibility tests among the patient with proper standardisation. A close association among the private laboratories and government organisations is clearly the need of the hour. We cannot afford to ignore this critical situation so that the control of tuberculosis in this subcontinent becomes highly jeopardised.

\section{CONCLUSION}

The study demonstrates the antitubercular drug-resistant pattern of the M. tuberculosis isolates in mostly urban population who were treated with various combination of first-line antitubercular drugs including the drug regimen prescribed by RNTCP. It will help us in our efforts of controlling drug resistance by modifying treatment regimen and other lacunae in the delivery system.

\section{REFERENCES}

[1] Murray CJ, Styblo K, Rouillion A. Tuberculosis in developing countries: burden, intervention, and cost. Bull Int Union Tuberc Lung Dis 1990;65(1):6-24.

[2] Snider DE, La Montagne JR. The neglected global tuberculosis problem: a report of the 1992 world congress on tuberculosis. J Infect Dis 1994;169(6):1189-96.
[3] Raviglione MC. Tuberculosis. In: Longo DL. (eds). Harrison's principles of internal medicine. 19th edn. Vol 2. New York. McGraw-Hill Education, 2015:1102-22.

[4] Wright A, Zignol M, Van Deun A, et al. Epidemiology of antituberculosis drug resistance 2002-07: an updated analysis of the global project on anti-tuberculosis drugs resistance surveillance. Lancet 2009;373(9678):186173.

[5] Woods GL. Mycobacteria. In: Henry JB. (eds). Clinical diagnosis and management by laboratory methods. $20^{\text {th }}$ edn. Philadelphia, WB Saunders Company, 2001:114457.

[6] Siddiqi SH. BACTEC 460TB system-Product and Procedure manual 1996.

[7] Laszlo A, Siddiqi SH. Evaluation of a rapid radiometric differentiation test for the mycobacterium tuberculosis complex by selective inhibition with p-nitro-alphaacetylamino-beta-hydroxypropiophenone. J Clin Microbiol 1984;19(5):694-8.

[8] Tenover FC, Crawford JT, Huebner RE, et al. The resurgence of tuberculosis: is your laboratory ready? J Clin Microbiol 1993;31(4):767-70.

[9] Hooker JA, Muhindi DW, Amayo EO, et al. Diagnostic utility of cerebrospinal fluid studies in patients with clinically suspected tuberculous meningitis. Int J Tuberc Lung Dis 2003;7(8):787-96.

[10] Roberts GD, Goodman NL, Heifets L, et al. Evaluation of the BACTEC radiometric method for recovery of mycobacteria and drug susceptibility testing of mycobacterium tuberculosis from acid-fast smearpositive specimens. J Clin Microbiol 1983;18(3):689-96. 